\title{
Intrusive luxation of tooth due to bite block after oral endotracheal intubation
}

\author{
Youn Kyung Son, Jae Moon Shin, Dong Ai An, and Yun Hong Kim
}

Department of Anesthesiology and Pain Medicine, Kangbuk Samsung Hospital, Sungkyunkwan University School of Medicine, Seoul, Korea

The most common legal claim that anesthesiologists can encounter after general anesthesia would be dental damage. The major causal factors for dental lesion, associated with oral endotracheal intubation, are patient's poor dentition, aggressive laryngoscopy, insufficient anesthesia and curarization, emergency interventions, and lack of experience by the anesthesiologist [1]. Differently from the above mentioned factors, authors report an experience of a dental injury caused by a bite block on a 5-year4-month-old boy with healthy looking primary teeth after endotracheal intubation.

A 5-year-4-month-old boy (height: $108.5 \mathrm{~cm}$, weight: 18.4 $\mathrm{kg}$ ) was admitted to the hospital for a surgical treatment on an ophthalmic diagnosis of exotropia and entropion of the eyelid. There were no abnormal findings on the preoperative assessment and dentition in particular.

When the patient's dental condition was assessed before the intravenous injection of anesthetic drug, there were still no abnormalities. To induce anesthesia, thiopental sodium 100 mg was intravenously administered, and after confirming his unconsciousness, a mixed solution of succinylcholine chloride 25 $\mathrm{mg}$ and atropine sulfate $0.1 \mathrm{mg}$ was administered and followed by sufficient muscular relaxation. After lifting the epiglottis toward the upper anterior region with rigid laryngoscope (Macintosh blade size 2), an overall view of the glottis was secured with Cormack \& Lehane grade 1, and then endotracheal intubation was performed. The blade of the laryngoscope did not touch any of the upper and lower incisors, and the uncuffed endotracheal tube 5.5 was easily inserted. Fixation of the tube was set at $16 \mathrm{~cm}$ with a bite block on the maxillary incisor. Bite block was used to prevent an airway obstruction caused by the patient biting the endotracheal tube unintentionally during anesthesia. Anesthesia was maintained with desflurane 6-7 vol\%, $\mathrm{O}_{2} 1 \mathrm{~L} / \mathrm{min}$, and $\mathrm{N}_{2} \mathrm{O} 1 \mathrm{~L} / \mathrm{min}$, and while atracurium 6 $\mathrm{mg}$ was intravenously given for muscle relaxation. Vital signs, $\mathrm{SpO}_{2}$, and $\mathrm{ETCO}_{2}$ during anesthesia were stable. After surgery, to reverse the effect of muscular relaxants, glycopyrrolate 0.1 $\mathrm{mg}$ and pyridostigmine $5 \mathrm{mg}$ were intravenously administered. As the patient was awakening from anesthesia, he started to bite a bite block attached to the endotracheal tube. Then, consciousness and spontaneous respiration were restored, while vital signs and $\mathrm{ETCO}_{2}$ were in the normal range and $\mathrm{SpO}_{2}$, was $100 \%$, thereafter the endotracheal tube was removed.

On postoperative one day, the patient complained of toothache and his parents found that the left lower incisor was intrusively luxated, approximately $1 \mathrm{~mm}$ compared with the right one. To exclude the possibility of a tooth fracture fragment being aspirated into the lung and stomach, a simple chest X-ray and abdomen $\mathrm{X}$-ray were taken. The results revealed no foreign bodies. When we referred him to the department of dentistry, it was confirmed that a mandible standard X-ray film (Fig. 1) showed the left lower incisor was luxated intrusively. The dental damage was identified to be a trauma created at the time of endotracheal intubation. It was not necessary for the intruded tooth to be restored because it was a milk tooth, but a treatment plan suggested that there should be a certain period of observation. One month later, when an $\mathrm{X}$-ray film of the mandible did not show any change, we decided

Corresponding author: Dong Ai An, M.D., Ph.D., Department of Anesthesiology and Pain Medicine, Kangbuk Samsung Hospital, Sungkyunkwan University School of Medicine, 108-1, Pyung-dong, Jongno-gu, Seoul 110-746, Korea. Tel: 82-2-2001-2319, Fax: 82-2-2001-2326, E-mail: andongai@gmail.com

(c) This is an open-access article distributed under the terms of the Creative Commons Attribution Non-Commercial License (http:// creativecommons.org/licenses/by-nc/3.0/), which permits unrestricted non-commercial use, distribution, and reproduction in any medium, provided the original work is properly cited. 


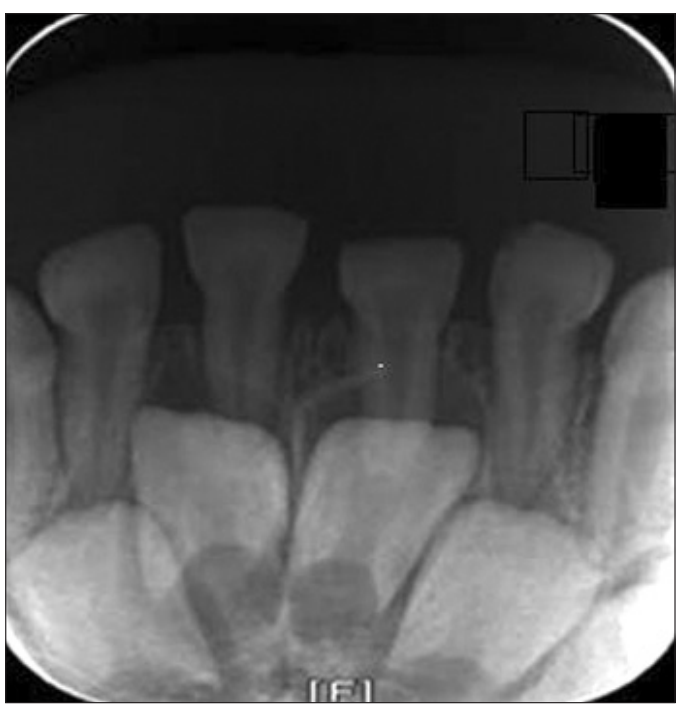

Fig. 1. Mandible standard radiograph 3 days after extubation shows that the left lower mandible milk tooth is intrusively luxated, compared with the other teeth.

to keep observing further.

Through preanesthetic assessment of patients, anesthesiologists should enquire about the patient's oral and dental conditions, including loose teeth, unstable crowns, any intraoral prostheses, and etc. Any preexisting poor dentition should be seen to a dental practitioner for the treatment. Dental damage ranges from loosening or facture of a tooth to displacement of a tooth from its socket. The incidences of dental injuries mostly occur to the maxillary incisors, and the upper left central incisor is particularly the most common site, damaged by the use of laryngoscope [2]

While various factors may contribute to the potential of dental damage during anesthesia, children in the ages of 6-8 years are in particular vulnerable to dental injuries at the time of oral endotracheal intubation because of prevalence of the immature roots, dilacerated roots, maxillary protrusion, and ectopic eruptions [3]. The child of the present case was 5-year-4-monthold boy with primary teeth, and did not seem to have had dental damage, at the time of the intubation.

Oropharyngeal airway or bite block is used to prevent airway obstruction caused by the patient biting the endotracheal tube unintentionally during anesthesia after the intubation. However, biting the devices tightly may have the potential to damage the teeth. Specially designed teeth guards may be used and an oropharyngeal airway or bite block can be employed with gauze rolls, in order to reduce dental damage. Fixing a bite block to the molar teeth can dissipate forces on the teeth throughout as many teeth as possible at the time of biting [3]. In this case, where the bite block was not wrapped in gauze or was not fixed to the molar teeth, could not exclude the potential of dental damage resulting from unintentional biting.

When dental injury occurs in children during oral endotracheal intubation, it is essential to consult with a pediatric dentist to determine the extent and the location of the damage, and to make an exact count of the damaged tooth fragments. If the retrieval of broken tooth fails, or any tooth fragments are missing, chest and abdomen X-ray are necessary to exclude aspiration into the lung or the stomach [4].

Most of dental injuries occur during endotracheal intubation by the use of a laryngoscope. Particularly, children in the age group of 6-8 years are susceptible to dental damage due to the above mentioned factors. Even a child with healthy-looking dentition may hurt one' own teeth by intensely biting the hard bite block accidently when emerging from anesthesia. Therefore, we strongly recommend fixing the bite block to the molar teeth or wrapping it in gauze at pediatric anesthesia to prevent any potential of dental damage.

\section{References}

1. Gaudio RM, Feltracco P, Barbieri S, Tiano L, Alberti M, Delantone M, et al. Traumatic dental injuries during anesthesia: part I: clinical evaluation. Dent Traumatol 2010; 26: 459-65.

2. Chen JJ, Susetio L, Chao CC. Oral complications associated with endotracheal general anesthesia. Ma Zui Xue Za Zhi 1990; 28 : 163-9.

3. Windsor J, Lockie J. Anaesthesia and dental trauma. Anaesth Intensive Care Med 2011; 12: 351-3.

4. Sowmya B, Raghavendra P. Management of dental trauma to a developing permanent tooth during endotracheal intubation. J Anaesthesiol Clin Pharmacol 2011; 27: 266-8. 